\title{
REMARKS ON TWO DETERMINANTAL INEQUALITIES
}

\author{
TIN-YAU TAM AND PINGPING ZHANG
}

Abstract. Denote by $\mathbb{P}_{n}$ the set of $n \times n$ positive definite matrices. Let $D=D_{1} \oplus \ldots \oplus D_{k}$, where $D_{1} \in \mathbb{P}_{n_{1}}, \ldots, D_{k} \in \mathbb{P}_{n_{k}}$ with $n_{1}+\cdots+n_{k}=n$. Partition $C \in \mathbb{P}_{n}$ according to $\left(n_{1}, \ldots, n_{k}\right)$ so that $\operatorname{Diag} C=C_{1} \oplus \ldots \oplus C_{k}$. For any $p \geqslant 0$, we have

$$
\operatorname{det}\left(I_{n_{1}}+\left(C_{1}^{-1} D_{1}\right)^{p}\right) \cdots \operatorname{det}\left(I_{n_{k}}+\left(C_{k}^{-1} D_{k}\right)^{p}\right) \leqslant \operatorname{det}\left(I_{n}+\left(C^{-1} D\right)^{p}\right) .
$$

This is a generalization of a determinantal inequality of Matic [6, Theorem 1.1]. In addition, we obtain a weak majorization result which is complementary to a determinantal inequality of Choi [2, Theorem 2] and ask a weak $\log$ majorization open question.

\section{Mathematics subject classification (2010): 15A45.} tion.

Keywords and phrases: Positive definite matrices, determinant, weak log majorization, weak majoriza-

\section{REFERENCES}

[1] R. Bhatia, Matrix Analysis, Springer, New York, 1997.

[2] D. CHOI, Determinantal inequalities of positive definite matrices, Math. Inequal. Appl., 19, (2016), $167-172$.

[3] R. A. Horn and C. R. Johnson, Matrix Analysis, Second Edition, Cambridge University Press, 2013.

[4] A. W. Marshall, I. Olkin, B. C. ARnold, Inequalities: Theory of Majorization and Its Applications, Second Edition, Springer, 2011.

[5] M. Lin, Determinantal inequalities for block triangular matrices, Math. Inequal. Appl., 18, (2015), 1079-1086.

[6] I. MATIC, Inequalities with determinants of perturbed positive matrices, Linear Algebra Appl., 449, (2014), 166-174.

[7] F. Zhang, Matrix Theory: Basic Results and Techniques, Second Edition, Springer, 2011.

[8] P. ZhANG, Extension of Matic's results, Linear Algebra Appl., 486, (2015), 328-334. 